INRA Prod. Anim., 2010, 23 (2), 111-122

\title{
Panorama mondial et européen de la production et de la consommation d'œufs
}

P. MAGDELAINE, A. BRAINE

Service Economie ITAVI, 4 rue de la Bienfaisance, F-75008 Paris, France Avec la collaboration de V. Gonnier, CNPO et de M.-P. Spiess, Ubifrance Courriel : magdelaine@itavi.asso.fr

Après une présentation du contexte international marqué par un développement de la production et des échanges mondiaux sur les dernières décennies, cet article analyse la situation et les enjeux de la filière européenne. Les évolutions du cadre réglementaire communautaire et les attentes des consommateurs conduisent à une diversification des systèmes de production, très variable selon les pays en raison de contextes nationaux différents. En conclusion, sont évoqués les facteurs d'évolution déterminant différents scénarios possibles pour les filières œufs française et européenne.

\section{1 / Panorama mondial}

1.1 / La production mondiale, dynamique et principaux pays producteurs

La production mondiale d'œufs s'est élevée à près de 61 millions de tonnes en 2008, selon la FAO, ce qui correspond à environ mille milliards d'œufs sur la base de 16,4 œufs par kg (Magdelaine 2009, Magdelaine et al 2010). La Chine, premier producteur mondial, représente à elle seule $37 \%$ de la production mondiale en 2008 suivie de l'Union Européenne à $27: 11 \%$, des Etats-Unis, de l'Inde et du Japon (figure 1).

La production mondiale a augmenté de $26 \%$ sur la dernière décennie, ce qui correspond à une croissance moyenne annuelle de $2,4 \%$, contre une croissance moyenne annuelle de 3,5\% sur la décennie 1988-1998, soit un léger ralentissement sur la dernière décennie par rapport à la décennie précédente.

La Chine notamment, dont la production avait augmenté très fortement dans les années 90, a enregistré un net ralentissement de cette croissance depuis 2000 (seulement $+2,6 \%$ par an de 1998 à 2008 , contre $+12 \%$ par an pendant la décennie précédente). D'autres pays asiatiques (Inde, Indonésie) ou le Mexique continuent d'afficher des taux de croissance annuels proches ou supérieurs à $5 \%$ (tableau 1 , figure 2 ).

\section{La production chinoise}

Dix provinces chinoises assurent $80 \%$ de la production d'œufs en 2006, contre $70 \%$ il y a 20 ans. Shandong et Hebei, en concentrent à elles seules, plus du

Figure 1. Production mondiale d'œufs en 2008 et évolution moyenne annuelle 1998-2008 (ITAVI d'après FAO et Commission européenne).

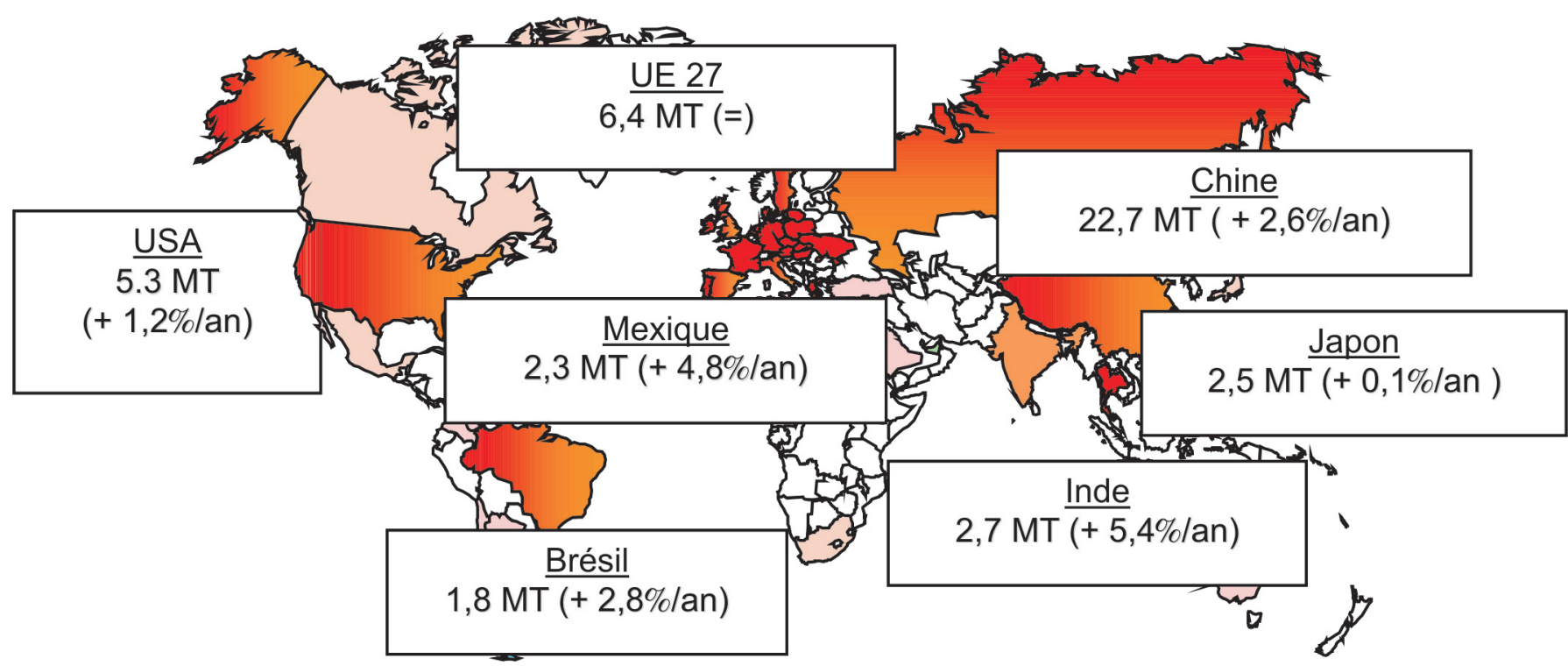


Tableau 1. Production d'œufs (de poules) dans le monde en tonnes (Source : FAO avril 2010).

\begin{tabular}{|l|c|c|c|c|c|}
\hline & 1988 & 1998 & 2008 & $\begin{array}{c}\mathrm{EMA}^{*}(\%) \\
1988-1998\end{array}$ & $\begin{array}{c}\mathrm{EMA}^{*}(\%) \\
1998-2008\end{array}$ \\
\hline Chine & 5738105 & 17531550 & 22749200 & 11,8 & 2,6 \\
UE-27 & 6718737 & 6716421 & 6539986 & 0,0 & $-0,3$ \\
USA & 4069000 & 4731000 & 5338700 & 1,5 & 1,2 \\
Inde & 1044000 & 1621000 & 2740000 & 4,5 & 5,4 \\
Japon & 2400061 & 2536035 & 2554000 & 0,6 & 0,1 \\
Mexique & 1090164 & 1461153 & 2337215 & 3,0 & 4,8 \\
Russie & & 1827930 & 2118500 & ND & 1,5 \\
Brésil & 1178359 & 1389539 & 1825000 & 1,7 & 2,8 \\
\hline Total Monde & $\mathbf{3 3 9 6 9 \mathbf { 4 7 7 }}$ & $\mathbf{4 8 0 8 9 4 9 3}$ & $\mathbf{6 0 6 7 8 3 9 1}$ & $\mathbf{3 , 5}$ & $\mathbf{2 , 4}$ \\
\hline
\end{tabular}

* EMA = Evolution Moyenne Annuelle.

** UE-27 reconstituée.

tiers. Hormis quelques régions d'élevages industrialisés, fournissant la grande distribution, situés à l'Est du pays (Shandong, Hebei...), les élevages chinois sont très atomisés, et tournés vers l'autoconsommation ou les marchés locaux. Un million d'éleveurs détiennent ainsi moins de 200 poules chacun et assurent à peine $10 \%$ de la production nationale, alors que les premiers producteurs (Dalian Hanwei, Beijing DQY) détiennent seuls ou sous contrat plus d'un million de poules. L'ensemble des pondeuses est élevé en cages, y compris dans les petits élevages.

Il n'existe pas de données récentes concernant la production d'ovoproduits ; en 2001, 5\% seulement de la production d'œufs étaient transformés. La consommation d'œufs chinoise est d'environ 333 œufs par personne et par an en 2008 (source International Egg Commission (IEC)) mais varie selon les années, les régions et les périodes de l'année. La consommation au Nord de la Chine est plus importante qu'au sud. L'épidémie d'influenza aviaire a fortement influencé la consommation d'œufs en 2005 et 2006. L'apaisement des médias sur ce sujet a permis de retrouver un meilleur niveau de consommation et de prix des œufs dès fin 2006. Les zones urbaines sont les plus fortes consommatrices d'œufs : Shanghaï, Beijing, Tianjin, Fujian, Guandong. Cependant, la consommation d'œufs frais en zones rurales augmente de manière régulière depuis quelques années, alors que la demande des consommateurs urbains tend à stagner et que les habitudes de consommation se diversifient.

\section{La production nord-américaine}

La production d'œufs aux Etats-Unis (3ème producteur mondial après la Chine et l'Union Européenne) s'élevait en 2008 à 5,3 MT, soit environ 87 milliards d'œufs. Sur les dix dernières années, la

Figure 2. Répartition de la production mondiale d'œufs (ITAVI d'après FAO).

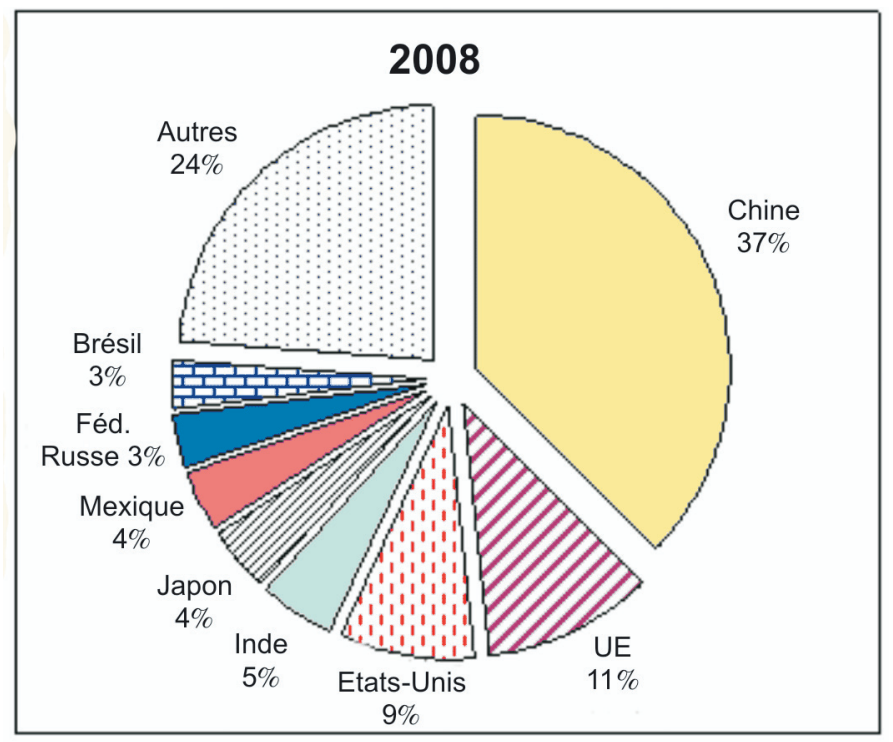

croissance de la production américaine a connu un développement plus soutenu que celui de la production européenne $(+1,2 \%$ par an contre $-0,3 \%$ par an pour l'UE).

Les structures de production aux USA sont très concentrées : 255 entreprises possèdent $95 \%$ des poules aux EtatsUnis (on dénombrait 284 millions de poules au 1 er février 2008). Six compagnies avec plus de 10 millions de pondeuses détiennent le tiers du cheptel nord-américain. Il n'existe pas aux USA de normes réglementaires de densité pour l'élevage des pondeuses. Cependant, une surface minimum de $430 \mathrm{~cm}^{2}$ par poule est considérée comme la densité de référence à partir de 2009. Le coût de production d'un œuf sortie élevage était estimé à 3,70 cents \$ en 2006 (Don Bell 2008, communication orale), soit environ 2,95 centimes d'euros, soit un coût inférieur de $35 \%$ au coût de production d'un œuf en cage en France en 2006.

Après un fort développement dans les années 1980 et 1990, la production d'ovoproduits tend à stabiliser sa part dans la production totale autour du tiers (31\% de la consommation totale en 2007, source IEC, Don Bell 2008).

La consommation globale d'œufs, très élevée dans les années 45-50 (autour de 380 à 400 œufs par personne et par an), a connu un long déclin pour atteindre 235 œufs par personne en 1995 et tend à se redresser depuis (248 œufs en 2008, source IEC).

\section{2 / Un commerce mondial dominé par les échanges intra- communautaires}

Les échanges mondiaux d'œufs et d'ovoproduits peuvent être estimés à partir des statistiques de la FAO. Les dernières données disponibles concernent 
Tableau 2. Principaux importateurs et exportateurs mondiaux d'œufs en coquille (Source : ITAVI d'après FAO).

\begin{tabular}{|l|c|l|c|}
\hline $\begin{array}{c}\text { EXPORTATEURS } \\
(\%)\end{array}$ & \multicolumn{2}{c|}{$\begin{array}{c}\text { IMPORTATEURS } \\
(\%)\end{array}$} \\
\hline Pays-Bas & 24 & Allemagne & 21 \\
Chine & 8 & Pays-Bas & 12 \\
Espagne & 7 & France & 12 \\
USA & 7 & Chine & 6 \\
Allemagne & 6 & Singapour & 4 \\
Malaisie & 5 & Royaume-Uni & 4 \\
\hline
\end{tabular}

l'année 2007. Les échanges internationaux ont porté en 2007 sur 1,4 million de tonnes d'œufs en coquille, $54800 \mathrm{~T}$ de poudre d'œufs et $217900 \mathrm{~T}$ d'ovoproduits liquides. En l'absence de détail sur les quantités d'ovoproduits échangées par nomenclature (part des entiers, des jaunes et des albumines), il est difficile d'estimer le volume global en «équivalent œuf coquille». Avec les coefficients de conversion utilisés pour transformer les ovoproduits entiers en tonnes «équivalent œuf coquille» (4,52 pour les œufs séchés et 1,17 pour les œufs liquides), on peut estimer ce volume global à 1,9 million de tonnes «équivalent œuf coquille», soit un peu moins de $3 \%$ de la production mondiale, commerce intra-communautaire inclus. Ces échanges internationaux sont globalement en augmentation sur les dix dernières années.

\section{a) Les échanges d'œufs en coquille}

Avec 1,4 million de tonnes d'œufs en coquille échangés en 2007 selon la FAO, en croissance de $63 \%$ sur les dix dernières années, le commerce mondial d'œufs en coquille représente près de $75 \%$ des échanges d'œufs et ovoproduits. Les principaux courants

Tableau 3. Principaux importateurs et exportateurs mondiaux d'œufs séchés (Source : ITAVI d'après FAO).

\begin{tabular}{|l|c|l|c|}
\hline \multicolumn{2}{|c|}{$\begin{array}{c}\text { EXPORTATEURS } \\
(\%)\end{array}$} & \multicolumn{2}{c|}{$\begin{array}{c}\text { IMPORTATEURS } \\
(\%)\end{array}$} \\
\hline Etats-Unis & 21 & Royaume-Uni & 16 \\
Inde & 14 & Allemagne & 11 \\
France & 13 & Japon & 10 \\
Pays-Bas & 12 & Danemark & 9 \\
Belgique & 7 & Pays-Bas & 6 \\
\hline
\end{tabular}

Tableau 4. Principaux importateurs et exportateurs mondiaux d'ovoproduits liquides (Source : ITAVI d'après FAO).

\begin{tabular}{|l|c|l|c|}
\hline \multicolumn{2}{|c|}{$\begin{array}{c}\text { EXPORTATEURS } \\
(\%)\end{array}$} & \multicolumn{2}{c|}{$\begin{array}{c}\text { IMPORTATEURS } \\
(\%)\end{array}$} \\
\hline Pays-Bas & 40 & Allemagne & 23 \\
France & 13 & France & 13 \\
Espagne & 8 & Royaume-Uni & 10 \\
Etats-Unis & 7 & Belgique & 10 \\
Allemagne & 6 & Danemark & 5 \\
\hline
\end{tabular}

Tableau 5. Apport protéique (g/personne/jour) (Source : FAO mars 2010).

\begin{tabular}{|l|l|l|l|}
\hline & $\mathbf{1 9 8 5}$ & $\mathbf{1 9 9 5}$ & $\mathbf{2 0 0 5}$ \\
\hline Viande de boeuf & 3,98 & 3,74 & 3,59 \\
œufs & $\mathbf{1 , 8 4}$ & $\mathbf{2 , 2 3}$ & $\mathbf{2 , 5 3}$ \\
Lait & 4,13 & 4,16 & 4,36 \\
Viande de porc & 3,41 & 4,05 & 4,4 \\
Viande de volaille & 2,28 & 3,3 & 4,33 \\
\hline
\end{tabular}

d'échanges se déroulent dans le cadre de l'Union Européenne (tableau 2).

\section{b) Les échanges d'ovoproduits}

Les échanges d'ovoproduits ont connu un développement important sur la dernière décennie. Ils ont en effet été multipliés par 1,8 pour les œufs séchés (54 $800 \mathrm{~T}$ en 2007 ) et par 1,6 pour les œufs liquides (217 $900 \mathrm{~T}$ en 2007).

Les six principaux exportateurs d'ovoproduits séchés assurent près de $72 \%$ des échanges mondiaux, avec une part croissante de l'Inde qui assure aujourd'hui $14 \%$ des exportations, contre $6 \%$ en 1997. La France se place en troisième position devant les Pays-Bas et la Belgique. Les importations sont également assez concentrées, six pays captant près de $55 \%$ des échanges. Les échanges d'ovoproduits liquides se déroulent, comme les échanges d'œufs en coquille, essentiellement dans le cadre communautaire (tableaux 3 et 4).

\section{3 / La consommation dans le monde et évolution sur les der- nières années}

Au niveau mondial, la consommation moyenne était estimée par la FAO à 9,1 $\mathrm{kg} /$ personne en 2005, soit environ 145 œufs. Les niveaux de consommation moyens varient fortement selon les pays : de plus de 300 œufs par personne au Japon, 230 à 240 œufs aux USA ou en Europe, à moins de 100 œufs par personne dans de nombreux pays africains ou d'Asie du sud-est.

Dans les pays en développement, la demande et la production d'œufs continueront à croître plus rapidement que dans les pays développés, en relation avec une croissance démographique plus forte et une consommation moyenne d'œufs par habitant fréquemment plus faible $(<100$ oeufs par an) que dans les pays développés.

Selon la FAO, les œufs sont, avec la viande de volaille, les produits ayant le plus accru leur contribution à la couverture des besoins protéiques de la population mondiale sur les deux dernières décennies (tableau 5).

Si le ratio apport protéique des œufs/apport protéique des produits laitiers se situe à 0,6 en moyenne, ce ratio varie largement selon les régions du monde de plus de 2 en Asie(5 en Corée, 3 en Thaïlande, 2,7 en Chine) à moins de 1 en Europe et en Amérique (1,1 au Canada, 0,9 en France, 0,5 en Allemagne et 0,4 aux Etats-Unis) et 0,2 au Brésil ou en Australie. 
Tableau 6. Principaux producteurs d'œufs de consommation de I'UE à 27 (ITAVI d'après le Service de la Statistique et de la Prospective (SSP) et la Commission Européenne).

\begin{tabular}{|l|c|c|c|}
\hline & $\begin{array}{c}\text { Production 2009 } \\
\text { (milliards d'oeufs) }^{*}\end{array}$ & $\begin{array}{c}\text { Evolution moyenne } \\
\text { annuelle 2000-2009 } \\
\mathbf{( \% )}\end{array}$ & $\begin{array}{c}\text { Evolution } \\
\mathbf{2 0 0 9 / 2 0 0 8}(\%)\end{array}$ \\
\hline France & 13,8 & $-0,7 \%$ & $-2,3 \%$ \\
Italie & 11,6 & $-1,5 \%$ & $-0,8 \%$ \\
Espagne & 11,4 & $-0,9 \%$ & $-2,0 \%$ \\
Allemagne & 9,9 & $-3,8 \%$ & $-17,1 \%$ \\
Royaume-Uni & 9,5 & $+0,2 \%$ & $+0,2 \%$ \\
Pays-Bas & 9,5 & $-0,4 \%$ & $+0,2 \%$ \\
Pologne & 8,8 & $+2,6 \%$ & $=$ \\
UE à 15 & 77,3 & $-1,3 \%$ & $-3,5 \%$ \\
10 NEM** & 18,4 & $+2,6 \%$ & $-1,9 \%$ \\
UE à 25 & 95,7 & $-0,7 \%$ & $-3,2 \%$ \\
Roumanie & 5,3 & nd & $=$ \\
Bulgarie & 1,4 & nd & $=$ \\
UE à 27 & 102,5 & nd & $-3,0 \%$ \\
\hline
\end{tabular}

* convertis sur la base de 16,4 œufs $/ \mathrm{kg}$.

${ }^{* *}$ NEM : Nouveaux Etats membres.

\section{2 / Panorama européen}

\section{1 / Stabilité de la production européenne depuis 2000}

La production de l'UE à 27 (2ème producteur derrière la Chine mais devant les Etats-Unis) était estimée par la Commission à 6,25 millions de tonnes en 2009 (soit un peu plus de 102 milliards d'œufs) en repli de $3 \%$ par rapport à 2008. Depuis 2000, la production de l'UE à 25 est presque stabilisée $(-0,6 \%$ par an), la production de l'UE à 15 enregistrant une diminution de $1,3 \%$ par an en moyenne, pendant que celle des dix nouveaux Etats membres (NEM) progressait de 2,6\% par an en moyenne en dépit d'un tassement en 2008 et en 2009 (tableau 6).

La France demeure le premier producteur de l'Union avec 13,8 milliards d'œufs produits en 2009. L'Italie arrive en seconde position suivie de l'Espagne avec environ 11,4 milliards d'œufs produits en 2009. Après une forte progression jusqu'en 2003-2004, la production espagnole marque cependant le pas depuis cing ans. Suivent l'Allemagne, en fort repli en 2009, le Royaume-Uni, les Pays-Bas et la Pologne (figure 3 ).

a) Les principaux pays producteurs européens (hors France)

L'Italie est le deuxième producteur européen d'œufs, derrière la France. Le marché italien se caractérise par une
$\mathrm{Au}$ sud de l'Italie, la production est beaucoup moins structurée, elle se trouve plutôt sous forme de petits élevages pour approvisionner un marché local. Les œufs produits étant essentiellement destinés à répondre à la forte demande en ovoproduits, ils sont majoritairement issus d'élevages en cages. Cependant, les consommateurs des régions au nord, plus proches des tendances sociétales suisses ou autrichiennes c'est-à-dire plus favorables au bien-être animal, commencent à s'intéresser aux œufs issus de systèmes de production alternatifs à la cage. Ces systèmes ont connu un développement récent pour atteindre $20 \%$ en 2008. La consommation globale d'œufs était de 224 œufs par personne et par an en 2008 .

L'Espagne qui avait fortement augmenté sa production d'œufs entre 2001 et 2004 est en net repli depuis, passant de 2 ème producteur européen d'œufs après la France en 2004 à la 3 ème place en 2009. Si le secteur des œufs y est encore peu concentré et très atomisé, la production se concentre peu à peu autour d'entreprises leaders. Les principales régions de production sont les deux Castilles et la Catalogne qui représentent à elles trois près de $50 \%$ de la production d'œufs. Le marché reste très peu segmenté en Espagne puisque 97\% des œufs produits sont issus d'élevages en cages. Les œufs plein air sont pour la majorité vendus hors de l'Espagne. 15\% des œufs sont transformés en ovoproduits. Environ $20 \%$ des œufs coquille sont exportés vers les autres pays de l’Union Européenne.

Les supermarchés sont devenus leaders dans la distribution des œufs pour les ménages suivis par le commerce traditionnel qui, malgré sa perte de parts

Figure 3. Principaux Etats membres producteurs d'œufs de consommation (ITAVI d'après SSP et Commission Européenne).

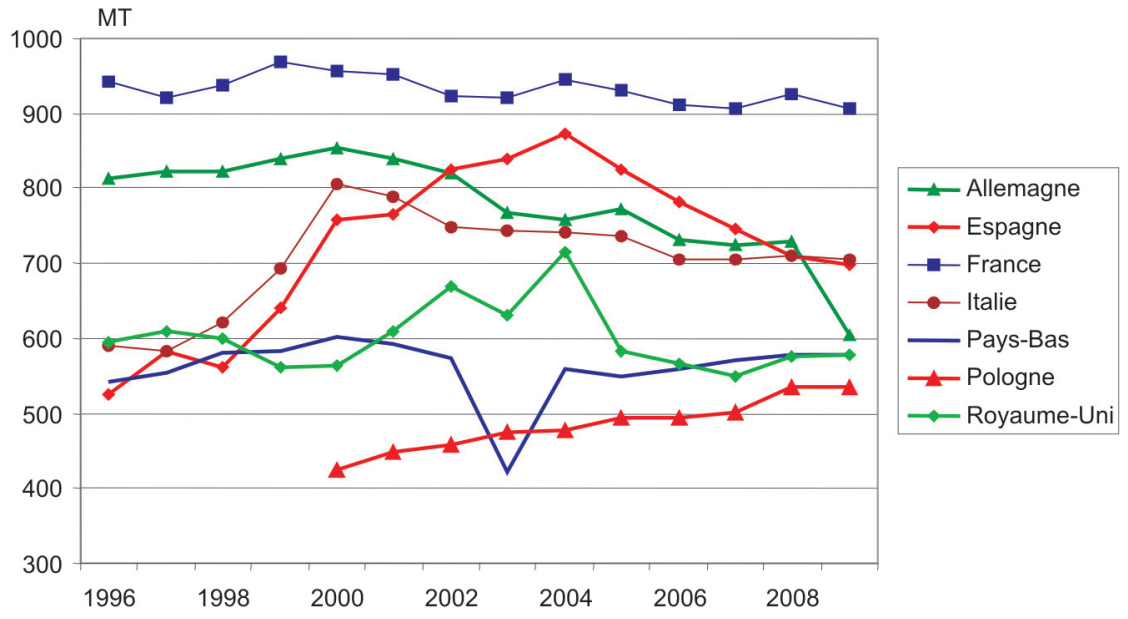


de marché, occupe encore une place non négligeable (30\% des achats en 2006). La consommation des œufs a chuté entre 1987 et 1995 essentiellement en raison des changements d'habitudes alimentaires. Depuis, elle s'est redressée pour se stabiliser à environ 189 œufs par personne et par an en 2008.

L'Allemagne est le quatrième producteur européen mais a la particularité de n'être autosuffisante qu'à $67 \%$ en 2008, voire seulement $57 \%$ en 2009 selon les premières estimations, en relation avec la baisse de production enregistrée l'an dernier. Elle est principalement approvisionnée par la filière hollandaise qui y commercialise les deux tiers de sa production.

Entre 1999 et 2007, les producteurs d'œufs étaient très incertains de leur avenir en raison de l'adoption d'une réglementation interdisant l'élevage en cages. La production en cages a d'ailleurs fortement diminué entre 1996 et 2006 pour atteindre $70 \%$ de la production contre 93\%, 10 ans auparavant et ce, en faveur des élevages plein air et au sol. Aujourd'hui, l'élevage en cages est interdit, et seuls les élevages en petits groupes (Klein Groupenhaltung) dans des grandes cages hautes hébergeant une soixantaine de poules, sont autorisés.

La Basse-Saxe, au nord de l'Allemagne, est la première région productrice où siègent les principales entreprises d'amont et d'aval de la filière. La concentration des productions avicoles et porcines dans cette région entraîne de fortes contraintes environnementales pour les éleveurs. La production est gérée par des entreprises qui intègrent, pour la plupart, plusieurs maillons de la filière. C'est le cas pour la première entreprise de production et de commercialisation d'œufs et d'ovoproduits, la Deutsche Frühstücksei. La consommation allemande est d'environ 212 œufs par personne et par an avec une tendance à la baisse entre 2000 et 2008.

La production au Royaume-Uni est également très concentrée. La première entreprise de production et de commercialisation des œufs et des ovoproduits, Noble Foods, a été créée à partir de la fusion des deux principaux leaders du marché britannique Deans Food et Stonegate. Pour faire face au déclin de la consommation suite à une crise médiatique sur l'œuf et la salmonelle fin des années 1980, la filière a mis en place un logo collectif de qualité : «British Lion quality» dans un objectif de réassurance du consommateur en imposant la vaccination des poules contre les salmonelles.
Actuellement, la consommation des ménages représente un peu moins de $45 \%$ de la consommation d'œufs, la Restauration Hors Domicile (RHD) près de $25 \%$ du marché et les ovoproduits $30 \%$ de la consommation britannique. Celle-ci se caractérise par une forte demande en œufs plein air qui représentent $30 \%$ du marché des œufs en 2007 contre environ $60 \%$ pour les œufs issus de poules en cages.

La Pologne est un des pays, parmi les nouveaux Etats membres ayant intégré l'Union Européenne, qui a le plus développé sa production d'œufs entre 1994 et 2004, elle en est d'ailleurs devenue exportatrice nette. La production se concentre dans deux régions centrales : la grande Pologne et la Mazovie.

La production d'œufs est très concentrée autour de quelques opérateurs (Wozniak, Pamira) même s'il reste encore une multitude de basses-cours. L'élevage en cages reste l'élevage dominant, toutefois, les élevages au sol et en plein air se développent. La filière apparaît très intégrée verticalement. La plupart des grandes exploitations possède leur propre centre d'emballage; le plus souvent, les producteurs ont des contrats à long terme spécifiant les volumes et la date de livraison (AGRA CEAS 2004).

La part des ovoproduits augmente mais elle reste faible comparée à celle des œufs coquille. Durant la deuxième moitié des années 90, le nombre d'entreprises de transformation des œufs a augmenté et le secteur de la transformation des œufs s'est fortement concentré : en 2003, on pouvait dénombrer 14 grandes entreprises, dont trois détenaient près de $95 \%$ du marché. Parmi elles, Ovopol, ancienne entreprise d'Etat, détient $75 \%$ des parts de marché.

b) Une diversification des modes d'élevage croissante au sein de l'Union Européenne

Depuis plus de dix ans, les systèmes d'élevage en Europe ont connu une forte diversification, en relation d'une part avec l'émergence de nouvelles attentes du consommateur et du citoyen, et d'autre part avec la mise en œuvre d'un cadre réglementaire concernant le bien-être des poules pondeuses. Ce cadre est défini au niveau communautaire par la Directive 99/74 /CE, traduite en droit français en février 2002. Cette directive définit des normes d'élevage à la fois pour les élevages en cages et pour les élevages alternatifs. Le point important pour les élevages en cages, est l'obligation de respecter certains aménagements des cages (nids, perchoirs et zones de grattage), ainsi qu'une superficie minimale de $750 \mathrm{~cm}^{2} /$ poule pour l'ensemble des élevages à partir de 2012. Des normes de commercialisation ont été définies également au plan communautaire dès 2001, concernant les appellations élevage en cages, au sol, et en plein air. Le mode de production biologique est défini par le règlement européen pour les productions animales biologiques (nouveaux règlements $834 / 2007$ et 889/2008 applicables au $1^{\text {er }}$ janvier 2009). L'indication du mode d'élevage sur les œufs par un code et en clair sur les boîtes est obligatoire depuis 2004.

Selon une compilation de données de la Commission européenne, et de l'ITAVI pour la France, 125 millions de pondeuses étaient élevées en systèmes alternatifs au sein de l'UE à 27 en 2008, soit environ $32 \%$ des effectifs de pondeuses européennes (tableau 7).

L'Allemagne est désormais le principal producteur d'œufs alternatifs au sein de l'UE avec 18 millions de pondeuses élevées hors des cages (40\% des effectifs), suivie par les Pays-Bas avec 17,1 millions de pondeuses alternatives en 2008 (55\% des effectifs nationaux), le Royaume-Uni et la France.

Au final, la diversification des systèmes d'élevage et la part des différents systèmes varient très fortement au sein de l’Union Européenne comme en témoigne

Tableau 7. Part des systèmes alternatifs au sein de l'UE à 27 : effectifs de poules pondeuses par système en 2008 (ITAVI d'après enquêtes et Commission européenne).

\begin{tabular}{|l|c|c|c|c|c|}
\hline & $\begin{array}{c}\text { Cage } \\
(\boldsymbol{\%})\end{array}$ & $\begin{array}{c}\text { Plein air } \\
(\boldsymbol{\%})\end{array}$ & Sol (\%) & $\begin{array}{c}\text { Biologique } \\
(\boldsymbol{\%})\end{array}$ & $\begin{array}{c}\text { Ensemble } \\
\text { des systèmes } \\
\text { alternatifs (\%) }\end{array}$ \\
\hline 1996 (UE à 15) & 92 & 4 & 4 & ND & 8 \\
2000 (UE à 15) & 89 & 6 & 5 & ND & 11 \\
2008 (UE à 27) & $\mathbf{6 8}$ & $\mathbf{1 7}$ & $\mathbf{1 3}$ & $\mathbf{2}$ & $\mathbf{3 2}$ \\
dont France & 81 & 12 & 3 & 4 & 19 \\
Espagne & 97 & 1 & 2 & - & 3 \\
Allemagne & 60 & 13 & 21 & 5 & 40 \\
Pays-Bas & 45 & 12 & 41 & 2 & 55 \\
Royaume-Uni & 48 & 36 & 8 & 5 & 52 \\
Italie & 80 & 1 & 16 & 2 & 20 \\
\hline
\end{tabular}


la figure 4. La part des systèmes alternatifs dans les effectifs de pondeuses est majoritaire en Autriche, en Suède et aux Pays-bas. Elle dépasse 35 à $40 \%$ au Royaume-Uni et en Allemagne et avoisine $20 \%$ en France, alors qu'elle demeure inférieure à $10 \%$ en Espagne et dans les nouveaux Etats membres. La France et le Royaume-Uni présentent une part plus importante des systèmes avec accès à un parcours extérieur.

Ce contexte réglementaire pourrait peser fortement à l'avenir sur la compétitivité de l'élevage européen. Les surcoûts liés à la seule prise en compte d'exigences de bien-être animal peuvent être estimés à partir des travaux de Peter Van Horne (Unversité de Wageningen (LEI)) et de l'ITAVI. Selon Peter Van Horne (2007, 2008), les coûts de production des œufs nord-américains ou brésiliens étaient en 2006 de 32 à 33\% inférieurs aux coûts européens. Les principaux facteurs de compétitivité nord et sud-américains résident dans le faible coût des intrants (matières premières pour l'alimentation animale et main-d'œuvre) et dans de plus faibles contraintes réglementaires notamment concernant le bien-être animal. Ce dernier facteur expliquerait 20 à $25 \%$ des écarts de coût de production avec l'Europe. La figure 5 visualise l'impact du seul critère bien-être animal (BEA).
Au sein de 1'Union Européenne, malgré un cadre communautaire commun, les contextes réglementaires nationaux diffèrent sensiblement et expliquent la diversité des situations constatées en terme de poids respectifs des différents systèmes d'élevage. Ainsi, les réglementations allemande et néerlandaise interdisent les cages même aménagées et n'autorisent qu'un système plus exigeant en termes d'espace disponible par poule (élevage en grand groupe dans des grandes cages ou mini-volières d'une hauteur minimale $60 \mathrm{~cm}$ de hauteur de cage et dans lesquelles les poules disposent de 800 à $900 \mathrm{~cm}^{2}$ par poule).

Figure 4. Part des systèmes alternatifs dans quelques Etats membres en 2008 (ITAVI d'après enquêtes et Commission européenne).

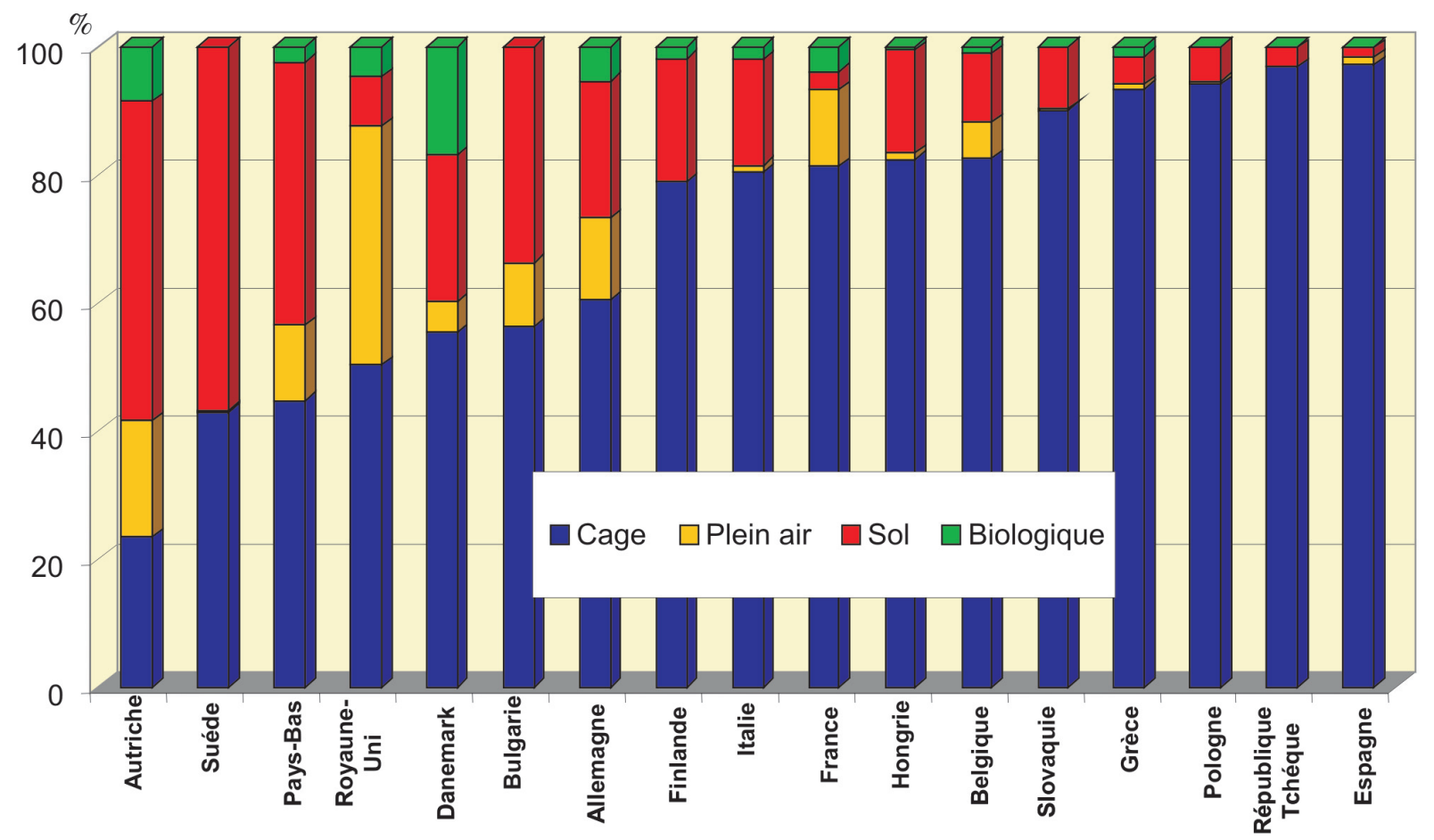

Figure 5. Le coût des contraintes bien-être animal (ITAVI d'après enquêtes et LEI).

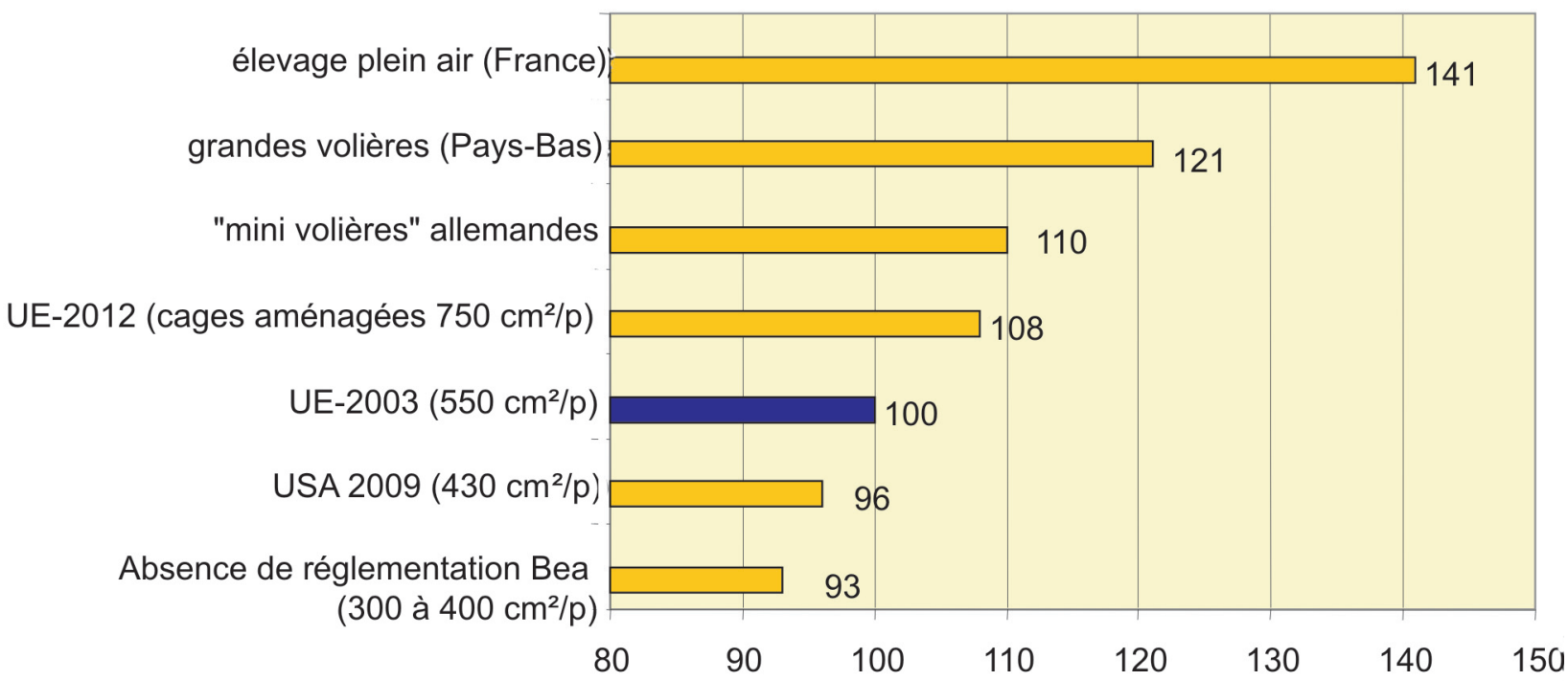

Base 100 : coût dans l'UE aux normes 2003 
Tableau 8. Principaux pays producteurs d'ovoproduits en Europe en 2005 (tonnes "équivalent œuf coquille») (Estimation ITAVI d'après SSP, IEC, Ubifrance et Statistiques nationales).

\begin{tabular}{|l|c|c|}
\hline & $\begin{array}{c}\text { Production 2005 en } \\
\text { T. Eq. OEuf coquille }\end{array}$ & $\begin{array}{c}\text { Part de la production } \\
\text { transformée (\%) }\end{array}$ \\
\hline France & 325000 & 36 \\
Italie & 280 à 300000 & 40 \\
Allemagne & 280000 & 38 \\
Pays-Bas & 152000 & 27 \\
Royaume-Uni & 132000 & 23 \\
\hline TOTAL UE- 25 & $\mathbf{1 5 0 0 0 0 0}$ & $\mathbf{2 4}$ \\
\hline
\end{tabular}

c) Près de $25 \%$ de la production européenne destinés aux ovoproduits

La production européenne d'ovoproduits est estimée à environ 1,5 MT en «équivalent œuf coquille» soit un peu moins du quart de la production européenne d'œufs. Les principaux producteurs sont la France et l'Italie, suivies par l'Allemagne, les Pays-Bas et le Royaume-Uni. L'Italie affiche la plus forte part d'œufs transformés en ovoproduits avec près de $40 \%$ de la production nationale (tableau 8).

\section{2 / Des échanges européens fai- blement excédentaires}

Les exportations extracommunautaires d'œufs et d'ovoproduits atteignent $185800 \mathrm{~T}$ en 2008 (en progression) dont un tiers d'œufs en coquille. Les importations extracommunautaires sont très faibles (23 $500 \mathrm{~T}$ «équivalent œuf coquille» en 2008).

Les statistiques communautaires concernant les échanges intracommunautaires d'œufs et d'ovoproduits ne sont plus publiés par la Commission européenne. Comme nous l'avons évoqué dans l'analyse des échanges internationaux, ils constituent une part

Tableau 9. Taux d'autosuffisance (\%) en œufs et de quelques pays européens (Source Zentrale Markt und Preisberichtstelle (ZMP) et ITAVI).

\begin{tabular}{|l|c|c|}
\hline & $\mathbf{1 9 9 8}$ & $\mathbf{2 0 0 8}$ \\
\hline Allemagne & 73 & 67 \\
France & 101 & 95 \\
Italie & 103 & 105 \\
Pays-Bas & 226 & 230 \\
Royaume-uni & 98 & 82 \\
Espagne & 102 & 113 \\
\hline UE - 15 & 103 & \\
\hline UE - 27 & & 101 \\
\hline
\end{tabular}

essentielle du négoce international et étaient estimés à $850000 \mathrm{~T}$ «équivalent ouf coquille» en 2002, au sein de l'UE à 15 , soit l'équivalent de 14 milliards d'œufs. Ils sont dominés par les exportations néerlandaises (taux d'autosuffisance de $220 \%$ en légère diminution sur les dix dernières années) et les importations allemandes qui affichent un taux d'autosuffisance assez stable entre 70 et 75\% (tableau 9).

\section{3 / Une consommation d'œufs et d'ovoproduits stabilisée en Europe, avec une forte segmen- tation des marchés}

Au sein de l'UE 27, la consommation alimentaire d'œufs et d'ovoproduits s'élève à environ 6,4 MT en 2008. Le niveau de consommation individuel moyen est d'environ $14,7 \mathrm{~kg}$ par habitant soit 240 œufs par personne et par an avec cependant d'importantes variations entre Etats membres, certains pays atteignant à peine 180 œufs par personne (Pays-Bas, Royaume-Uni), alors que d'autres, comme le Danemark, atteignent presque 300 œufs. Deux tendances communes se (ITAVI d'après IEC et Statistiques nationales). dégagent cependant de l'analyse de la consommation au sein de l'UE : la part croissante des ovoproduits dans la consommation globale d'une part, et le développement de la part des œufs alternatifs dans la consommation d'œufs en coquille à domicile (hors restauration hors domicile) d'autre part. Selon les pays, la segmentation du marché de l'œuf coquille et la part des œufs issus des différents systèmes d'élevage est très variable (tableau 10, figure 6).

La part des différents systèmes d'élevage dans la production de chaque pays (tableau 7) diffère sensiblement de celle des différents types d'œufs dans la consommation d'œufs en coquille (tableau 10). Ainsi, aux Pays-Bas, 45\% des œufs sont produits en cages, alors que seulement $11 \%$ de la consommation intérieure d'œufs en coquille sont constitués d'œufs en cages. En effet, d'une part une partie des œufs produits sont exportés et ne se retrouvent donc pas dans la consommation intérieure du pays; d'autre part, une part de la consommation est faite sous forme d'ovoproduits non inclus dans la consommation d'œufs en coquille (figure 6).

\section{3 / La filière ouf française}

\section{1 / Une production française stabilisée sur les quatre derniè- res années}

La production française a connu une période de croissance dans les années 90 , avec près de 16 milliards d'œufs produits en 1999. Depuis, elle est globalement orientée à la baisse, malgré une augmentation sensible en 20042005. Elle a induit une situation de surproduction et des difficultés importantes sur le marché et a eu une légère reprise en 2008.

Tableau 10. La consommation d'œufs dans différents Etats membres en 2008

\begin{tabular}{|l|c|c|c|}
\hline & $\begin{array}{c}\text { Consommation } \\
\text { 2008 (CEufs/hab.) }\end{array}$ & $\begin{array}{c}\text { Part des } \\
\text { ovoproduits } \\
\mathbf{( \% )}\end{array}$ & $\begin{array}{c}\text { Part des œufs alternatifs } \\
\text { dans la consommation } \\
\text { des ménages (volume) } \\
\text { (\%) }\end{array}$ \\
\hline Hongrie & 251 & nd & nd \\
France & 248 & 31 & 29 \\
Italie & 224 & 33 & $<10$ \\
Belgique & 184 & 50 & nd \\
Allemagne & 212 & nd & 61 \\
Espagne & 189 & nd & $<5$ \\
Pays-Bas & 182 & 22 & 89 \\
Royaume-Uni & 183 & 20 & 37 \\
Danemark & 300 & 42 & nd \\
Suède & 197 & 19 & 63 \\
\hline
\end{tabular}


Figure 6. Part des différents types d'œufs dans la consommation d'œufs en coquille dans différents Etats membres en 2007 (ITAVI d'après Nielsen, BEIC (British Egg Industry Council), Gfk (Institut d'étude de la consommation et de la distribution), ZMP).

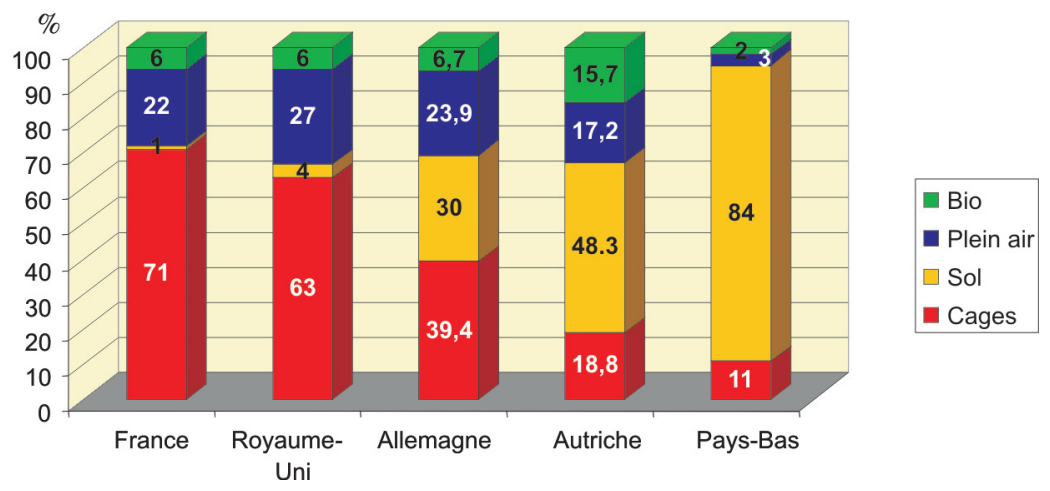

La production française tend à se diversifier depuis la fin des années 80 . Les effectifs de poules pondeuses élevées en système alternatif sont estimés à 8,3 millions de poules en 2008 , soit $18,7 \%$ du cheptel de pondeuses en France. Les effectifs estimés en 2008, à partir des données de l'agence Bio, du SYNALAF, de l'ITAVI et de la DGAL sont les suivants : 1,7 millions de pondeuses bio, 1,6 millions de pondeuses Label Rouge (LR), 3,6 millions de pondeuses plein air hors LR et 1,3 million de pondeuses au sol. Les productions les plus dynamiques sur les cinq dernières années sont celles d'œufs sous label rouge et d'œufs biologiques dont les taux de croissance annuelle atteignent en moyenne sur 5 ans respectivement 4 et $2,6 \%$. La production alternative en France se caractérise par la prédominance des systèmes avec parcours et la faible part de la production au sol en claustration, dont la production est destinée essentiellement à 1'exportation (figure 7).

Figure 7. Part des différents systèmes d'élevage en France en 2008 (Estimation ITAVI d'après SSP, DGAL, SYNALAF, Agence BIO et enquêtes auprès des opérateurs).

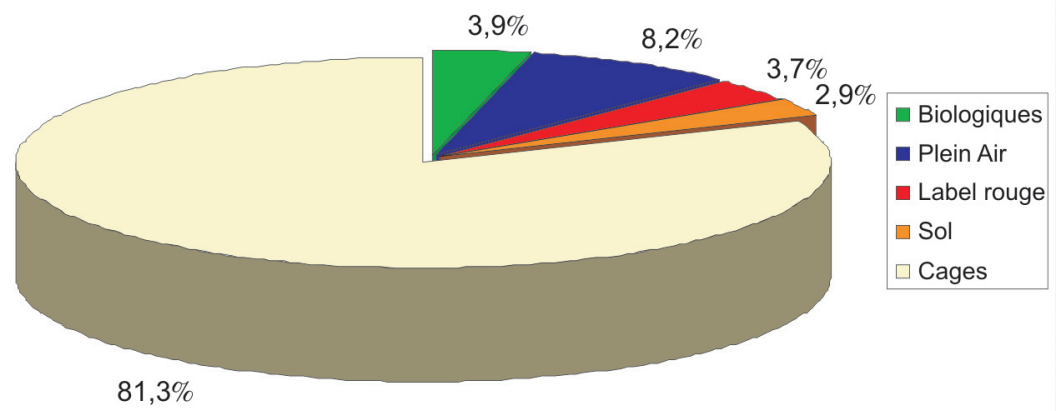

de production (46000 pondeuses par exploitation en moyenne pour la production en cages contre 7000 pondeuses pour la production de plein air). Les exploitations détenant des pondeuses en cages représentaient $44 \%$ des exploitations pondeuses, mais assuraient $82 \%$ des livraisons d'œufs et détenaient $80 \%$ des capacités de production, alors que le système plein air ( $\mathrm{y}$ compris label rouge) représentait $36 \%$ des exploitations pour seulement $9 \%$ des livraisons, et la production biologique $14 \%$ des exploitations pour $3 \%$ des livraisons.

Les éleveurs travaillent, soit sous contrat avec une organisation de production, le plus souvent liée à un fabricant d'aliment, de type coopératif ou privé, soit de façon indépendante pour les plus importants d'entre eux qui possèdent leur propre centre d'emballage. Ces structures de taille plus importante ou fermes de ponte (comptant une ou plusieurs centaines de milliers de poules) peuvent elles-mêmes s'approvisionner, pour partie, auprès de plus petits producteurs avec qui elles passent un contrat d'approvisionnement.

b) Les centres d'emballage et les industriels en ovoproduits

500 centres d'emballage sont recensés par les services statistiques du Ministère de l'Agriculture. Le nombre de centres a fortement progressé au début des années 2000, avec la multiplication du nombre des fermes de ponte qui disposaient d'avantages logistiques dans l'approvisionnement des zones de consommation. Depuis, la mise en place de plates-formes d'approvisionnement par la grande distribution et la recherche d'économies d'échelle conduisent à une inversion de tendance et à une forte concentration du secteur. Les centres d'emballage sont le plus souvent regroupés au sein de groupes commerciaux nationaux qui ont mutualisé leur fonction marketing et qui négocient avec les centrales d'achat de la grande distribution.

Environ soixante industriels produisent des produits à base d'œufs à destination de l'industrie agroalimentaire (biscuiterie, charcuterie ; traiteur, pâtes alimentaires, sauces, entremets et desserts) ou de la RHD.

\section{3 / Une segmentation croissan- te des marchés, une reprise du marché de l'œuf coquille en 2008 et 2009}

Depuis le 1er janvier 2006, en raison d'un changement de méthodologie du Service des douanes, les échanges en volume d'œufs de consommation, ne peuvent plus être analysés. Cependant 
Figure 8. Répartition de la consommation globale d'œufs et d'ovoproduits en 2008 (Elaboration ITAVI d'après SCEES et Douanes).

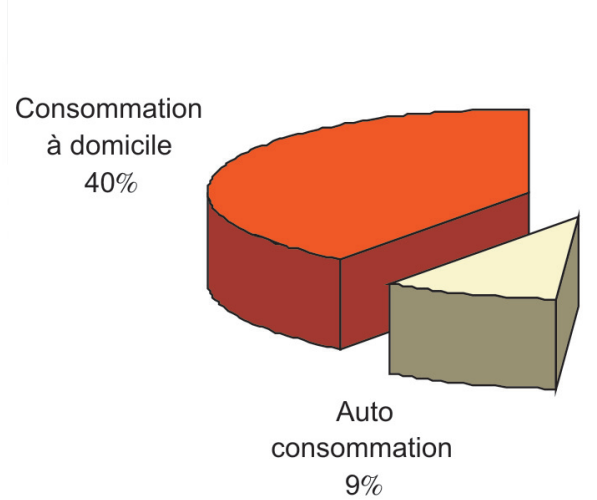

des estimations faites par l'ITAVI sur les volumes échangés, à partir des prix moyens européens, ont permis de calculer un taux d'autosuffisance estimé à $98 \%$ en 2008 . Cependant, le solde de nos échanges en valeur est excédentaire de 31,7 M€ en 2008.

L'ITAVI a également réalisé une estimation de la consommation intérieure globale en 2006, 2007 et 2008. Celle-ci s'établit à environ 248 œufs par personne en moyenne sur les trois dernières années.

La consommation nationale se répartit entre 9\% d'autoconsommation (œufs produits et consommés sur l'exploitation), $40 \%$ d'achats des ménages pour la consommation à domicile via différents circuits de distribution, 20\% d'achats d'œufs en coquille par la RHD, et $31 \%$ d'utilisation de produits transformés (ovoproduits) par les industries alimentaires (biscuiterie pâtisserie, pâtes alimentaires, sauces, entremets desserts...) et la RHD. La part des ovoproduits dans la consommation globale d'œufs se développe régulièrement, passant de 22\% en 1997 à

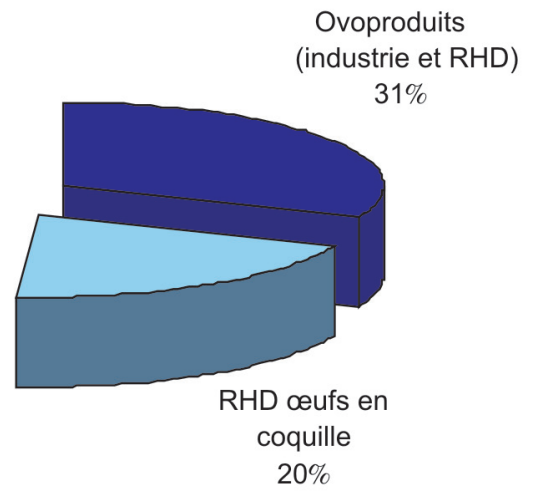

$31 \%$ en 2007 ; elle pourrait atteindre près de $40 \%$ de notre consommation globale d'œufs d'ici dix ans. Les attentes des consommateurs pour l'achat d'œufs en coquille sont diverses et laissent une place croissante aux œufs alternatifs qui représentent près de $30 \%$ des achats en volume et plus de $40 \%$ des ventes d'œufs en valeur dans les Grandes et Moyennes Surfaces (GMS). $\mathrm{La}$ restauration et l'industrie privilégient prix et qualité sanitaire, ce qui se traduit par une part assez faible des œufs alternatifs sur ces marchés (figure 9).

Les ventes d'œufs en coquille en GMS représentent selon les panels distributeurs un peu moins de 4 milliards d'œufs, soit environ un tiers de la consommation globale hors autoconsommation. Ce marché est caractérisé par une part croissante des segments «œufs alternatifs» dans les linéaires. Depuis 2004, en France, les ventes d'œufs en GMS (hyper et supermarchés) étaient en recul en valeur malgré une légère progression en volume. Depuis 2007, on assiste à un rebond du marché à la fois en volume et en valeur.
Cette reprise du marché est portée essentiellement par les segments d'œufs alternatifs (bio, label rouge et plein air) qui représentent aujourd'hui environ $30 \%$ des volumes vendus en GMS, et $45 \%$ du chiffre d'affaires du rayon œufs.

Sur l'ensemble de l'année 2009, les ventes d'œufs LR progressent de 9,3\% en volume et de $6,8 \%$ en valeur, celles d'œufs biologiques progressent de $17,5 \%$ en volume et de $18,9 \%$ en valeur et les ventes d'œufs plein air de 10,4\% en volume et $9,1 \%$ en valeur, alors que les ventes totales d'œufs progressent de $5 \%$ en volume et $5,7 \%$ en valeur (figure 9).

\section{Conclusion}

Les perspectives de développement demeurent favorables sur le marché mondial, tiré par la demande de pays émergents comme la Chine, l'Inde ou le Mexique. La situation est plus stable en Europe et en France, avec des marchés qui se segmentent selon deux axes, d'une part au profit du développement de la production d'ovoproduits, qui atteint près du quart de la production européenne d'œufs, d'autre part au profit des systèmes de production alternatifs, qui rassemblent $25 \%$ du cheptel européen de poules pondeuses, et qui représentent près de $30 \%$ de la consommation d'œufs en coquille au sein de l'Union. Ces développements demeurent cependant très hétérogènes entre Etats membres (Magdelaine et al 2010).

Les différents facteurs d'évolution qui détermineront les scénari possibles pour l'avenir sont les suivants (Magdelaine et Jez 2009) :

- Le contexte réglementaire communautaire lié au bien-être animal. La

Figure 9. Segmentation du marché des œufs en GMS en 2009 (hyper + supermarchés) (Source Census IRI d'après Matines).
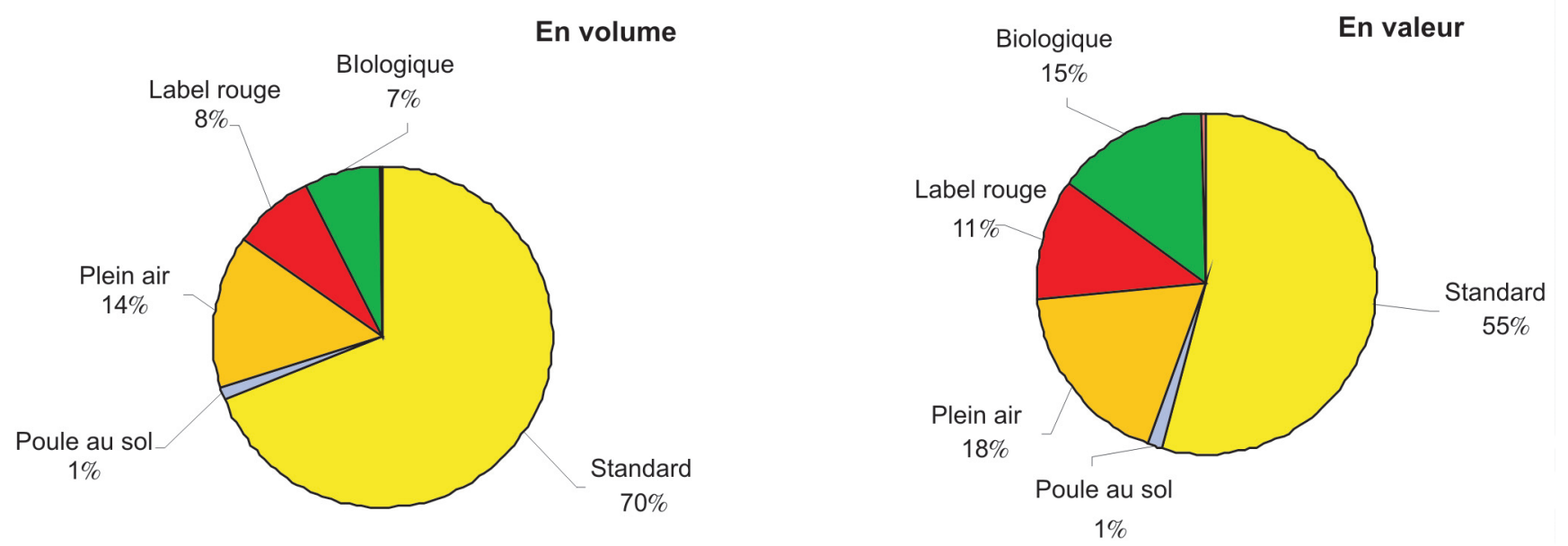
récente confirmation par la Commission européenne, de l'échéance du 1er janvier 2012 pour l'interdiction des cages non aménagées devrait accentuer la diversification des systèmes d'élevage et un développement des systèmes alternatifs ;

- Des exigences également croissantes en matière de protection de l'environnement et de sécurité sanitaire et de lutte antisalmonelles, qui pourraient alourdir les coûts de production ;

- Une forte volatilité des prix des matières premières entrant dans l'alimentation animale et des incidences fortes sur les évolutions des prix de revient ;

- Des tendances d'évolution des comportements d'achat des consommateurs et des modes de consommation encore incertaines : d'une part, la crise économique renforce certainement la sensibilité au prix, ce qui est favorable à la consommation d'œuf, qui reste la protéine la moins chère; d'autre part, une part croissante des consommateurs est sensible aux modes d'élevage et d'ali- mentation des animaux, comme en témoigne la forte demande en produits biologiques. Ces nouvelles attentes des consommateurs sont par ailleurs relayées, voire anticipées par les acteurs de la grande distribution ;

- Des négociations à l'Organisation Mondiale du Commerce (OMC), qui pourraient déboucher sur une ouverture accrue du marché communautaire, et supprimer les protections douanières existantes.

Dans ce contexte, les acteurs des filières française et européenne doivent réaliser un effort d'adaptation des outils de production très important d'ici 2012 et effectuer des choix stratégiques d'investissement. Ces investissements les engageront pour les vingt prochaines années (cages aménagées, minivolières, grandes volières), alors que les modèles techniques restent à tester voire à améliorer sur de nombreux points. Le développement des productions alternatives devra se faire à un rythme compatible avec les évolutions de la demande et éviter des surproductions conjoncturelles (à l'instar de ce qu'ont connu les Pays-Bas récemment avec un fort développement de la production en volières, un engorgement du marché de l'œuf «volière» et une baisse des prix payés aux producteurs).

Enfin, les systèmes de production de demain devront garantir à la fois la sécurité sanitaire des produits, le respect du bien-être animal et des prix compétitifs afin d'assurer l'approvisionnement de la RHD et de l'industrie $(50 \%$ de la consommation aujourd'hui, peut être plus de $60 \%$ en 2020). Cette exigence de compétitivité sera d'autant plus forte dans un contexte d'ouverture probable du marché européen aux importations en provenance de pays tiers ne supportant pas les mêmes contraintes. Une diminution de la protection tarifaire communautaire est en effet probable dans le cadre des négociations à l' $\mathrm{OMC}$, alors qu'il sera vraisemblablement difficile d'exiger des normes minimales en matière de bien-être animal dans ces négociations internationales.

\section{Références}

AGRA CEAS, 2004. Consulting study on the socio economic implications of the various systems to keep laying hens. Contract SANCO /2003/ SPC, 2003258.

Directive 1999/74/CE du Conseil du 19 juillet 1999 établissant les normes minimales relatives à la protection des poules pondeuses. JOCE L 203/53 du 3 août 1999.

Don Bell, 2008. Conférence WG1 de la WPSA Paris, France, Juin, Communication orale.

Magdelaine P., 2009. Future prospects for the European egg industry. XIII Eur. Symp. Quality of Eggs and Egg Products, 21-25 June 2009, Turku Finland, WPSA, Finish branch, PL3.pdf.

Magdelaine P., Jez C., 2009. Conclusions de la prospective réalisée par l'ITAVI et l'INRA sur l'aviculture française à l'horizon 2025.
Journ. ITAVI pondeuse. Ploufragan, 3 décembre. ITAVI (Ed), Paris, France.

Magdelaine P., Braine A., Gonnier V., Spiess M.P., 2010. Production et consommation des oufs et ovoproduits. In : Science et technologie de l'œuf et des ovoproduits. Nau F., GuérinDubiard C., Baron F., Thapon J.L. (Eds) Editions Tec et Doc Lavoisier, Paris, France, 1, 1-36.

Règlement CE n ${ }^{\circ} 834 / 2007$ du Conseil du 28 juin 2007 relatif à la production biologique et à l'étiquetage des produits biologiques et abrogeant le règlement (CEE) $n^{\circ} 2092 / 91$. JOUE L 189 du 20.07.2007.

Règlement CE $\mathrm{n}^{\circ} \quad 889 / 2008$ de la Commission du 05/09/2008 portant modalités d'application du règlement (CE) $n^{\circ} 834 / 2007$ du Conseil relatif à la production biologique et à l'étiquetage des produits biologiques en ce qui concerne la production biologique, l'éti- quetage et les contrôles. JOUE L $250 \mathrm{du}$ 18.09.2008.

Van Horne P., 2007. Prohibition of enriched cages for laying hens in the Netherlands? An examination of the consequences. LEI report 2.07.10, Agricultural Economics Research Institute. The Hague. August 2007.

Van Horne P., 2008. Production costs of table eggs; an international comparison. LEI report 2008-071. Agricultural Economics Research Institute. The Hague. December 2008.

\section{www.agreste.agriculture.gouv.fr}

www.circa.europa.eu

www.fao.org

www.internationalegg.com

\section{Résumé}

La production européenne d'œufs a atteint 6,4 MT en 2008, soit un peu plus de $10 \%$ de la production mondiale. Si production et consommation mondiales demeurent en croissance sur la dernière décennie, la production européenne se stabilise et connaît une forte diversification des systèmes d'élevage, en relation avec une évolution de la demande des consommateurs mais aussi en conséquence de la mise en ouvre de la directive bien-être. La situation au sein de l'UE demeure contrastée selon les Etats membres. La consommation européenne est de $\mathbf{2 4 0}$ oufs par personne et par an en moyenne, avec une part croissante des ovoproduits. Différents facteurs d'évolution détermineront les scénari possibles pour l'avenir, parmi lesquels le contexte réglementaire communautaire (bien-être animal, protection de l'environnement, exigences sanitaires), la forte volatilité des prix des matières premières entrant dans l'alimentation animale, l'évolution des comportements d'achat des consommateurs et des modes de consommation, et enfin l'issue des négociations à l'OMC qui pourraient déboucher sur une ouverture accrue du marché communautaire, et supprimer les protections douanières existantes. 


\begin{abstract}
International and European overview of egg production and consumption

Egg production in the EU-27 reached 6.4 MT in 2008, which is a little more than $10 \%$ of world production. Even though world egg production and consumption has been growing for the ten last years, egg production in the EU has been stable in volume and production systems have known a very strong evolution in relation with consumer expectations in terms of animal welfare and variety of products, but also due to the implementation of the $E U$ welfare regulation. The situation remains contrasted within the EU. European egg consumption is 240 eggs per person per year, with strong variations according to country, and a rise in the share of egg products in global consumption. Different steering factors will determine the future of the European egg industry, among which European regulation (animal welfare, environment, sanitary requirements), feedstuff price volatility, changes in consumers' preferences and lastly the conclusions of WTO negotiations, which could lead to an opening of the European market to products (egg powder for example) from emerging countries.
\end{abstract}

MAGDELAINE P., BRAINE A., 2010. Panorama mondial et européen de la production et de la consommation d'œufs. In : Numéro Spécial, Qualité de l'œuf. Nys Y. (Ed). Inra Prod. Anim., 23, 111-122. 